\title{
How Common are Habitable Planets?
}

Jack J. Lissauer

Space Science Division, MS 245-3, NASA Ames Researç Center, Moffett Field, CA 94035

lissauer@ringside.arc.nasa.gov

The Earth is teeming with life, which occupies a diverse array of environments; other bodies in our Solar System offer fewer, if any, niches which are habitable by life as we know it. Nonetheless, astronomical studies suggest that a large number of habitable planets are likely to be present within our Galaxy. 


\section{How Common are Habitable Planets?}

One of the most basic questions that has been pondered by Natural Philosophers for many millennia concerns humanity's place in the Universe: Are we alone? This question has been approached from a wide variety of viewpoints, and similar reasoning has led to widely diverse answers. Copernicus, Kepler, Galileo and Newton convincingly demonstrated that other worlds qualitatively similar to Earth orbit the Sun. In the past few years, two dozen planets have been discovered in orbit about stars other than our Sun.

The intellectual and technological advances of the past century leave us poised at the turn of the millennium to investigate the possibility of extraterrestrial life along numerous paths of experimental, observational and theoretical studies in both the physical and life sciences. The conventional, very anthropocentric, picture is that to be habitable, a planet must have liquid water on its surface for a very long time. This single factor is unlikely to be necessary or sufficient. Nonetheless, I will accept it as a definition in order to make the astronomical problem cleanly posed.

\section{STELLAR PROPERTIES AND HABITABLE ZONES}

Stars are huge balls of plasma which radiate energy from their surfaces and liberate energy via thermonuclear fusion reactions in their interiors. During the star's long-lived main sequence phase, hydrogen in its core is gradually "burned up" to maintain sufficient pressure to balance gravity. The star's luminosity grows slowly during this phase, as fusion increases the mean particle mass in the core and greater temperature is required for pressure balance. Once hydrogen in the core is used up, the star's structure and luminosity change much more rapidly. Sun-like and larger stars expand and become red giants; stars initially $>\sim 8$ times the Sun's mass can end their lives in spectacular supernova explosions.

The main sequence phase of low mass stars provides "continuously habitable zones", where planets may maintain liquid water on their surfaces for billions of years. High mass stars are much hotter than low mass stars, and use up their fuel far more rapidly. Thus, even if Earth-like planets form around high mass stars at distances where liquid water is stable, it is unlikely that benign conditions exist long enough on these planets for life to form and evolve. However, the greater flux of ultraviolet radiation may speed up biological 
evolution enough to compensate for a moderately massive star's shorter lifetime. At the other end of the spectrum, the smallest, faintest stars can live for trillions of years, but they emit almost all of their luminosity at infrared wavelengths and their luminosity varies by tens of percent due to flares and large starspots. Also, habitable zone planets orbit so close to these faint stars that their rotation is tidally synchronized (as the Moon's is relative to Earth); thus no day-night cycle occurs, and if the planet's atmosphere is thin it freezes out on the perpetually dark, cold, hemisphere (Joshi et al. 1997).

\section{STABILITY OF PLANETARY SYSTEMS}

Although isolated single planet systems are stable essentially indefinitely, mutual gravitational perturbations within multiple planet systems can lead to collisions and ejections. To first approximation the star's gravity dominates, but planets exchange orbital energy and angular momentum, so that over millions or billions of orbits, even weak perturbations can move planets out of benign orbits. Resonances among various orbital and precession frequencies are the major source for chaos in planetary systems. There aren't any simple criteria for determining the stability of systems with many planets, but in general large spacing between orbits, small eccentricities and inclinations, and low mass planets are more stable (Lissauer 1999).

The bottom line for habitable planets is this: The minimum separation of Earth mass planets on low eccentricity orbits required for the system to be stable for long periods of time is comparable to the width of a star's continuously habitable zone. Thus, orbital stability arguments support the possibility that most stars could have one or even two planets with liquid water on their surfaces, but unless greenhouse effects conspire to substantially compensate for increasing distance from the star, larger numbers of habitable planets per system are unlikely.

\section{PLANET FORMATION}

Stars are observed to be forming within cold regions of the galaxy called molecular clouds. Even a very slowly rotating molecular cloud core of stellar mass has far too much angular momentum to collapse down to an object of stellar dimensions, so a significant fraction of the material in a collapsing core falls onto a rotationally-supported 
disk orbiting the pressure-supported (proto)star. Such a disk has the same initial elemental composition as the growing star. At sufficient distances from the central star, it is cool enough for $\sim 1-2 \%$ of this material to be in solid form, either remnant interstellar grains or condensates formed within the disk. The growth from micron-sized dust to kilometer-sized planetesimals remains poorly -understood (Weidenschilling and Cuzzi 1993).

Kilometer-sized and larger planetesimals in protoplanetary disks travel on elliptical orbits that are altered by mutual gravitational interactions and physical collisions. These interactions lead to accretion (and in some cases erosion and fragmentation) of planetesimals. Gravitational encounters stir planetesimal random velocities up to the escape speed from the largest common planetesimals in the swarm (Safronov 1969). Sufficiently massive and dense planets far enough from the star can hurl material into interstellar space. Oort Cloud comets are believed to be icy planetesimals which were sent outwards at nearly the Solar System escape velocity, and were perturbed into long-lived orbits by close stars, interstellar material or the galactic tidal field.

Giant planet growth times predicted by current models (Pollack et al. 1996) are similar to estimates of the lifetime of gaseous protoplanetary disks. Thus, giant planets might not form in most protoplanetary disks. Although giant planets themselves are unlikely abodes for life, they may harbor habitable moons. Moreover, they affect both the stability of terrestrial planet orbits and the flux of impactors impinging on terrestrial planets (Wetherill 1994). Such impacts can have a devastating effect on life - a fact that no dinosaur is likely to argue with.

The gravitational pull of known extrasolar planets induce velocity variations in their stars much larger than would a planetary system like our own, and surveys accomplished to date could not detect low mass and long period planets (www.gmarcy). Our own Solar System may represent a biased sample of a different kind, because it contains a planet with conditions suitable for life to evolve to the point of being able to ask questions about other planetary systems (Wetherill 1994). Unfortunately, we do not now have the capability to develop planet formation models from first principles, nor even from observations of protostellar disks, whose detailed properties are poorly constrained. However, theory suggests that a great of diversity of planetary systems are possible (Lissauer 1995). 


\section{IMPACTS, DINOSAURS AND THERMOPHILES}

The largest mass extinction of the past $\sim 200$ million years occurred 65 million years ago, when roughly half of genera of multicellular organisms, including all of the dinosaurs, suddenly died. off. The geological record shows a layer of impact-produced minerals and iridium, an element rare in Earth's crust but more abundant in primitive meteorites, deposited at the time that the dinosaurs vanished. Additionally, the largest known crater on Earth dated at less than one billion years old was formed at this time. Taken together, these data imply that the K-T mass extinction was caused by the impact of $\mathrm{a} \sim 10 \mathrm{~km}$ radius asteroid or comet into the Yucatan peninsula (Chapman and Morrison 1994).

Impacts, like earthquakes, come in various sizes, with the large ones much rarer but vastly more hazardous than the small ones (Table 1). Because of the destruction that impacts may produce, impact frequency is an important factor in planetary habitability. The impact rate on the terrestrial planets of our Solar System was orders of magnitude larger four billion years ago than it is at present. In a planetary system like our own but with smaller planets replacing Jupiter and Saturn, large impact fluxes could continue, making planets with earthlike compositions and radiation fluxes hostile abodes for living organisms (Wetherill 1994).

\section{PLANETARY PROPERTIES AND HABITABILITY}

Life on Earth has been able to evolve and thrive thanks to billions of years of benign climate. Mars appears to have had a climate sufficiently mild for liquid water to have flowed on its surface when the Solar System was roughly one-tenth its current age, but at the present epoch, the low atmospheric pressure means that liquid water is not stable on the martian surface. Venus is too hot, with a massive carbon dioxide dominated atmosphere; we cannot say whether or not young Venus had a mild Earth-like climate. Indeed, as stellar evolution models predict that the young Sun was about $25 \%$ less luminous than at present, we don't understand why Earth, much less Mars, was warm enough to be covered by liquid oceans 4 billion years ago.

Carbon dioxide on our planet cycles between the atmosphere, the oceans, life, fossil fuels and carbonate rocks on a wide range of 
timescales. The carbonate rocks form the largest reservoir; they are produced by reactions involving water (and in some cases living organisms). Carbon dioxide is recycled from carbonates back into the atmosphere as plates are subducted and heated within the Earth's mantle. Carbonates are not readily recycled on a geologically inactive planet such as Mars; in contrast they are not formed on planets like Venus, which lack surface water. Larger planets of a given composition remain geologically active for longer, as they have smaller surface area to mass ratios, and thus retain heat from accretion and radioactive decay longer. The number of variables involved in determining a planet's habitability precludes a complete discussion, but some of the major issues are summarized in Figure 1 (cf. Lewis 1998).

\section{INTERSTELLAR EAVESDROPPING}

The Search for ExtraTerrestrial Intelligence (SETI) is an endeavor to detect signals from alien life forms (www.seti). A clear detection of such a signal would likely change humanity's world view as much as any other scientific discovery in history. As our society is in its technological infancy, another civilization capable of communicating over interstellar distances is likely to be enormously advanced compared to our own -- compare our technology to that of a mere millennium ago and then extrapolate millions or billions of years into the future! Thus, a dialog with extraterrestrials could alter our society in unimaginable ways (and they could probably answer most if not all of the questions raised in this article).

The primary instrument used by SETI is the radiotelescope. Most radio waves propagate with little loss through the interstellar medium, and many wavelengths also easily pass through Earth's atmosphere. They are easy to generate and to detect. Radio thus appears to be an excellent means of interstellar communication, whether data are being exchanged between a community of civilizations around different stars or broadcast to the galaxy in order to reach unknown societies in their technological infancy. Signals used for local purposes, such as radar and TV on Earth, also escape and can be detected at great distances.

The first deliberate SETI radiotelescope observations were performed by Frank Drake in 1960. Since that time, improvements in receivers, data processing capabilities and radiotelescopes have doubled the capacity of SETI searches roughly every 8 months. 
Nonetheless, only a minuscule fraction of directions and frequencies have been searched, so one should not be discouraged by the lack of success thus far.

\section{A DELUGE OF DATA TO COME}

The speculation about advanced civilizations on Mars that was rampant a century ago has been quashed by better observations. However, evidence for a wet Mars billions of years ago suggests that life might have formed on that planet, and microbes may still be present below the surface (McKay 1997). Interplanetary spacecraft will soon attempt to determine whether or not life ever existed on Mars, and if so what were/are its characteristics (mpfwww). NASA plans to investigate a possible subsurface ocean in Jupiter's moon Europa (www/fire_ice). The Cassini mission en route to Saturn will study the properties of Titan's atmosphere (www.cassini). Although Titan is too cold for liquid water, this large moon has a methane-rich atmosphere, in which photochemical reactions create organic molecules. Analogous processes may have occurred within the Earth's early atmosphere.

Interstellar probes for in situ exploration of extrasolar planets require substantial technological advances. Even with gravity assists by the planets and the Sun, payloads sent with current rocket propulsion systems would require thousands of years to reach the nearest stars. Thus at present, interstellar travel remains in the realm of science fiction, but considering the advances of the past millennium, voyages over such vast distances may become practical in the upcoming centuries.

Although we are not yet able to reach the stars, we are nonetheless entering a golden age of extrasolar planet studies via telescopic observations. About two dozen extrasolar planets are now known. All of these objects have been identified indirectly, via the gravitational force that they exert on their star, and all have been found during the 1990's. The first two extrasolar planets to be discovered orbit a rapidly spinning neutron star, which emits a substantial fraction of its luminosity in the $x$-ray region of the spectrum. All of the extrasolar planets known to orbit main sequence stars are more massive than Saturn. They were discovered using the Doppler technique, which measures changes in the radial velocity of the star in response to the planet's gravitational tug. 
Various techniques should immensely increase our knowledge of extrasolar planets in the upcoming decades. Doppler studies should continue to find giant extrasolar planets (www.gmarcy). Current precision is sufficient to detect Jupiter mass planets in Jupiter-like orbits and Uranus mass planets orbiting very close to their stars. Better precision may eventually lead to identification of smaller and more distant planets, but turbulence and other variability of stellar atmospheres will make detection of Earth analogs via the Doppler technique impractical if not impossible.

Planets may also be detected via the wobble that they induce in the motion of their stars projected into the plane of the sky. This astrometric technique is most sensitive to massive planets orbiting about stars which are relatively close to Earth. Because the star's motion is detectable in two dimensions, a better estimate of the planet's mass can be obtained than using radial velocities. No astrometric claim of detecting an extrasolar planet has yet been confirmed, but technological advances (especially the development of optical/IR interferometry) suggest that jovian mass and possibly smaller planets will be detected from the ground using this technique within the next decade. Even higher precision is likely to be achievable from spacecraft observations. The ultimate limit to astrometric precision is likely to be differences between the positions a star's center of mass and its center of light which result from starspots and other brightness variations.

If Earth lies in or near the orbital plane of an extrasolar planet, that planet passes in front of the disk of its star once each orbit as viewed from Earth. Precise photometry can reveal such transits, which can be distinguished from rotationally-modulated starspots and intrinsic stellar variability by their periodicity, and would provide the size and orbital period of the detected planet. Scintillation in and variability of Earth's atmosphere limit photometric precision to roughly one thousandth of a magnitude, allowing detection of transits by Jupiter-sized planets but not by Earth-sized planets from the ground (www.vulcan). Far greater precision is achievable above the atmosphere, with planets as small as Earth likely to be detectable (www.kepler). This technique has the greatest potential for detecting Earth analogs within the upcoming decade.

Distant planets are very faint objects which are located near much brighter objects (the star or stars that they orbit), and thus they are extremely difficult to image. Efforts are currently underway to 
directly image giant planets from the ground using adaptive optics and coronagraphs. NASA and ESA are both currently designing space missions for the second decade of the 21 st century that will use interferometry and nulling to search for Earth-like planets around nearby stars (www.darwin). Assuming such planets are detected, spectroscopic investigations of their atmospheres, to search for gases such as oxygen, ozone and methane, will follow. Obtaining resolved images of the disks of extrasolar earths requires substantially better optics and much greater light gathering capability, but unlike interstellar travel, such distant resolved images should be achievable within the 21 st century.

Observations of planetary formation provide information on properties of planets as a class, and typical impact fluxes that terrestrial planets are exposed to. The infrared region of the spectrum are likely to provide the greatest information about star and planet formation in the upcoming decades because planetary regions radiate most of their energy in the mid-IR and dusty interstellar clouds transmit far more scattered starlight in the nearIR than in the visible. Advances in infrared detectors, interferometry and new large telescopes on the ground with adaptive optics and in space (www.ngst) will provide vastly superior data to those available today.

\section{PROSPECTUS}

Prognostications have a miserable success rate. Forecasts centuries into the future tend to be particularly conservative. In part, this reflects deficiencies in the human imagination. However, the average rate of change greatly exceeds the median rate. Consider the implication of joining the galactic club of civilizations a billion years more advanced than our own (assuming such a community exists!). Such a revelation would lead to changes far more fundamental than the invention of movable type, the industrial revolution and the information age have brought to us within the past millennium.

We still do not know whether terrestrial planets on which liquid water flows are rare, are the norm for solar type stars or have intermediate abundances. Nonetheless, I personally believe that planets with liquid water oceans lasting for long periods of time are sufficiently common that if we are the only advanced life form in our sector of the galaxy, biological factors are much more likely to be the principal limiting factor than are astronomical causes. 
Figure 1

A smaller planet made of the same material as Earth would be less dense, because the pressure in the interior would be lower. Such a planet would have a larger ratio of surface area to mass, so its interior would cool faster. Its lower surface gravity and more rigid crust would allow for higher mountains and deeper valleys than are seen on Earth. Most important to life is that it would have a much smaller surface pressure as a result of four factors: larger surface area to mass, lower surface gravity, more volatiles sequestered in crust since less crustal recycling and more atmospheric volatiles escaping to space. This would imply, among other things, lower surface temperature, because less greenhouse gases in the atmosphere.

Some remedial measures which could improve the habitability of such a mass-deprived planet are:

1) Move it closer to the star, so less greenhouse effect would be needed to keep surface temperatures comfortable.

2) Add extra atmospheric volatiles.

3) Include a larger fraction of long-lived radioactive nuclei than on Earth, to maintain crustal recycling.

A larger planet made of the same material as Earth would be denser and have a hotter interior. Its higher surface gravity and less rigid crust would lead to muted topography. It would have a much greater atmospheric pressure, and, unless its greenhouse was strong enough to boil away the planet's water, much thicker oceans, probably covering the planet's entire surface.

Some remedial measures which could improve the habitability of such a mass-gifted planet are:

1) Move it farther from the star.

2) Include a smaller fraction of atmospheric volatiles.

It isn't clear that more active crustal recycling would be a problem, within limits. 


\section{Impacts \& Life}

\begin{tabular}{|c|c|c|c|c|}
\hline Size & Example(s) & Most Recent & \multicolumn{2}{|c|}{ Planetary Effects Effects on Life } \\
\hline $\begin{array}{l}\text { Super Colossal } \\
\mathrm{R}>2000 \mathrm{~km}\end{array}$ & Moon-forming event & $4.45 \times 10^{9} \mathrm{yr}$ ago & Melts planet & $\begin{array}{l}\text { Drives off volatiles } \\
\text { Wipes out life on planet }\end{array}$ \\
\hline $\begin{array}{l}\text { olossal } \\
>700 \mathrm{~km}\end{array}$ & $\begin{array}{l}\text { Pluto } \\
1 \text { Ceres (borderline) }\end{array}$ & $>\sim 4.3 \times 10^{9} \mathrm{yr}$ ago & Melts crust & Wipes out life on plane \\
\hline $\begin{array}{l}\text { Imbo } \\
>200 \mathrm{~km}\end{array}$ & $\begin{array}{l}4 \text { Vesta } \\
\text { (large asteroid) }\end{array}$ & $\sim 4.0 \times 10^{9} \mathrm{yr}$ ago & \multicolumn{2}{|c|}{$\begin{array}{ll}\text { Vaporizes oceans } & \begin{array}{l}\text { Life may survive } \\
\text { below surface }\end{array}\end{array}$} \\
\hline $\begin{array}{l}\text { xtra Large } \\
>70 \mathrm{~km}\end{array}$ & $\begin{array}{l}\text { Chiron } \\
\text { (largest active comet) }\end{array}$ & $3.8 \times 10^{9} \mathrm{yr}$ ago & \multicolumn{2}{|c|}{ 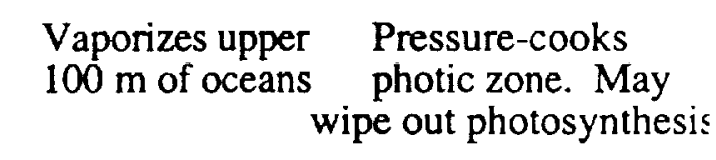 } \\
\hline$>30 \mathrm{~km}$ & Comet Hale-Bopp & $\sim 2 \times 10^{9} \mathrm{yr}$ ago & \multicolumn{2}{|c|}{$\begin{array}{l}\text { Heats atmosphere Continents cauterizes } \\
\text { and surface to } \sim 1000 \mathrm{~K}\end{array}$} \\
\hline $\begin{array}{l}\text { Medium } \\
\mathrm{R}>10 \mathrm{~km}\end{array}$ & $\begin{array}{l}\text { K-T impactor } \\
\text { Eros (largest NEA) }\end{array}$ & $6 \mathrm{x} \times 10^{6} \mathrm{yr}$ ago & \multicolumn{2}{|c|}{$\begin{array}{l}\text { Fires, dust, darkness; Half s } \\
\text { atmosphere/ocean } \\
\text { chemical changes, } \\
\text { large temperature swings }\end{array}$} \\
\hline mall & ～500 NEA's & $\sim 300,000$ yr ago & $\begin{array}{l}\text { Global dusty } \\
\text { atmosphere } \\
\text { for months }\end{array}$ & $\begin{array}{l}\text { Photosynthesis inter- } \\
\text { rupted, individuals die } \\
\text { but few species extinct, } \\
\text { civilization threatened }\end{array}$ \\
\hline $\begin{array}{l}\text { ewee } \\
>100 \mathrm{~m}\end{array}$ & Tonguska event & 91 years ago & \multicolumn{2}{|c|}{$\begin{array}{l}\text { Major local effects Newspaper headline: } \\
\text { Minor hemispheric Romantic sunsets } \\
\text { dusty atmosphere increase birth rate }\end{array}$} \\
\hline
\end{tabular}


Table legend

The effects of an impact on life depend in a qualitative way on the impact energy. The smallest space debris to hit Earth's atmosphere is slowed to benign speeds by gas drag or vaporized before it hits the ground. The largest impactors can melt a planet's crust and eliminate life entirely. Strong iron impactors ranging in size from a car to a house may hit the ground at high velocity, killing living beings in their path. The rocky bolide that exploded oyer Tonguska, Siberia in 1908 was about the size of a football field; it produced a blast wave which knocked over trees tens of kilometers away. An impactor a few kilometers in size would throw enough dust into the upper atmosphere that it could substantially darken the sky for much of a growing season (Toon et al. 1997); the threat of such an impactor to human food supplies has led NASA to initiate a program to detect all near-Earth asteroids (NEA's) larger than $\sim 1 \mathrm{~km}$. Mass extinctions result from even larger impacts, which load the atmosphere with dust and chemicals (from vapor and pulverized matter originating in both the impactor and the crater ejecta) and radiation from high-velocity ejecta reentering the atmosphere may cause global fires. Even larger impacts fill the atmosphere with enough hot ejecta and greenhouse gases to vaporize part or all of the planet's oceans (Sleep et al. 1989). Indeed, phylogenetic evidence implies that the last common ancestor of all life on Earth was a thermophilic prokaryote, which would have been most capable of surviving such a scalding impact. Even larger impacts would destroy all life on the planet, although it is possible that some organisms could survive in meteoroids ejected by the impact, and subsequently reestablish themselves on the planet (or another planet orbiting the same star) by a fortuitously gentle return after conditions on the planet had improved. 